\title{
The influence of immunosuppression on apoptosis and necrosis during experimental trichinellosis in mice
}

\author{
JOLANTA PIEKARSKA ${ }^{1}$, MICHAE GORCZYKOWSKI', MARIANNA SZCZYPKA ${ }^{2}$, \\ BOŻENA OBMIŃSKA-MRUKOWICZ2
}

${ }^{1}$ Department of Internal and Parasitic Diseases, Faculty of Veterinary Medicine, Wroclaw University of Environmental and Life Sciences, Poland

2Department of Biochemistry, Pharmacology and Toxicology, Faculty of Veterinary Medicine, Wroclaw University of Environmental and Life Sciences, Poland

\begin{abstract}
A study was carried out to determine the influence of immunosuppressive drug on apoptosis and necrosis lymphocytes in the spleen and mesenteric lymph nodes of mice experimentally infected with Trichinella spiralis. CFW mice were infected with 200 larvae of Trichinella spiralis. Dexametasone was injected intra-peritoneally, at a dose of $25 \mathrm{mg} / \mathrm{kg}$ body weight, ten times at 24 hour intervals (between 1-5 and 18-22 day of infection). The lymphocytes obtained from the spleen and mesenteric lymph nodes on 7, 14, 21, 28, 35, 42 and 60 day after infection were incubated using Annexin-V-Fluos Staining Kit. Results of the study demonstrated that dexametasone increased the percentage of apoptotic lymphocytes in the spleen and mesenteric lymph nodes from 14 day after infection in mice infected with T. spiralis and decreased the percentage of the necrotic lymphocytes in both examined lymphatic organs.
\end{abstract}

Key words: T. spiralis, apoptosis, necrosis, immunosuppresion, dexametasone, mice.

(Centr Eur J Immunol 2012; 37 (3): 204-208)

\section{Introduction}

Trichinellosis is a zoonotic infection caused by the parasite nematodes belonging to the genus Trichinella. Pathological changes during infection are closely connected with the development of two generations of Trichinella spiralis in the intestine and in the muscle tissue. In the intestinal phase of trichinellosis a protective response is induced in the mucosal immunological system. Inflammatory infiltrations developing in the intestine after infection with $T$. spiralis have features of allergic reactions [1]. T-helper (Th) 2-type lymphocytes have a significant participation in modulating immunological reaction. Both Th1 cells as well as Th2 cell type response is in lymphatic organs but in the spleen Th1 lymphocytes are more active. However, the Th2 are more active in mesenteric lymph nodes [2,3]. Although they do not directly participate in the elimination of the parasite, they determine the type, the intensity and the speed of the immune response via secreted cytokines $[4,5]$.
Expulsion of T. spiralis from intestine is associated with synthesis of specific IgE, mastocytosis prolonged under IL-4 and eosinophilia induced by IL-5. The intestinal muscle hypercontractility and goblet cells hyperplasia appear as a result of infection-induced immune-mediated changes in intestinal physiology which is associated with the worm expulsion [6]. Parasites developed a lot paths in order to avoid the immune response of the host and one of them is arousing the apoptosis of effector cells [7]. It was demonstrated that excretory/secretory antigens of helmints trigger the apoptosis in subpopulation of lymphocytes in lymphatic organs and can contribute to existence the immunosuppression and finally to the longer survival in the body of the host. It is also not possible to exclude their participation in triggering necrosis which leads to rapid disintegration of a cell with all consequences of this process. The apoptosis is regarded as the natural death of cells which ensures the homeostasis, so this kind of death is more

Correspondence: Jolanta Piekarska, Department of Parasitology, Faculty of Veterinary Medicine, Wroclaw University of Environmental and Life Sciences, Norwida 31, 50-375 Wrocław, Poland, e-mail: jolanta.piekarska@up.wroc.pl 
favourable. T. spiralis invasion induces apoptosis of some inflammatory infiltration cells in the lamina propria of intestinal mucosa and also in muscle tissue [8]. In mice infected with T. spiralis and subjected to actions of immunostimulators, an increase of percentage of apoptotic lymphocytes and decrease of percentage of necrotic lymphocytes in spleen and mesenteric lymph node lymphocytes was observed. However, no significant impact of these processes was demonstrated on the number of parasites in intestines and muscles [9]. The aim of the current study was to detect apoptosis and necrosis among lymphocytes of spleen and mesenteric lymph nodes in mice infected with T. spiralis under condition of immunosuppression.

Glucocorticoids are used in the treatment of early stage of trichinellosis for the reason that they suppress the symptoms of immediate-type hypersensitivity. They show the anti-inflammatory and immunosuppressive activity through inhibition the activity of immune cells (including lymphocytes) and through inducing the changes in population together with decreasing cytokine synthesis $[10,11]$.

\section{Material and methods}

The study was carried out on $126 \mathrm{CFW}$ inbred mice. The mice were about three months old, and weighed about 20 grams. 84 mice were orally infected with $200 \mathrm{~T}$. spiralis larvae, T1(ISS1820 Poland). The larvae used in the infection were recovered from the muscle tissue of mice infected 2-3 months earlier by digestion with $1 \%$ pepsin $/ \mathrm{HCl}$ solution for $4 \mathrm{~h}$ at $37^{\circ} \mathrm{C}$.

Dexametasone (Dexaven Jelfa, series 011005) was administered intra-peritoneally, at a dose of $25 \mathrm{mg} / \mathrm{kg}$ body weight, ten times at 24 hour intervals. The first set of five doses of dexametasone (DEX) was administered starting on the $1^{\text {st }}$ day of infection, and the second set of five doses was administered starting on the $18^{\text {th }}$ day of infection.

The mice were divided into three experimental groups: - Control: mice infected with T. spiralis,

- $\mathrm{DEX}^{+}$: mice infected with $T$. spiralis and treated with DEX,

- DEX: uninfected mice treated with DEX.

All mice were maintained under identical conditions for the duration of the experiment.

The study protocol was approved by the Local Ethics Committee in Wroclaw, Poland (No 29/2008).

\section{Isolation of lymphocytes}

Six mice from each group were sacrificed using halothane (Narcotan, Leciva) on days 7, 14, 21, 28, 35, 42 and 60 after infection. The spleen and mesenteric lymph nodes were removed aseptically, transferred to sterile phospho-buffered saline (PBS) at $4^{\circ} \mathrm{C}$ and pressed through a nylon cell strainer to produce a single cell suspension. Lymphocytes were isolated by Ficoll 400/ Uropolinum 75\% density - gradient in centrifugation for $15 \mathrm{~min}$ at $4^{\circ} \mathrm{C}$ [12].
Cells collected from the interphase were washed twice with PBS with $1 \%$ bovine serum albumin (BSA; Sigma) at $4^{\circ} \mathrm{C}$. For spleen samples, this fraction was treated with $0.84 \%$ $\mathrm{NH}_{4} \mathrm{Cl}$ in order to disrupt erythrocytes. After a second wash, the cells were adjusted to $1 \times 10^{6} \mathrm{cell} / \mathrm{ml}$. Cell viability was determined by staining with trypan blue. The proportion of viable lymphocytes was greater than $96 \%$.

\section{Measurement of apoptosis and necrosis}

Annexin V-Fluos Staining Kit (Roche-Diagnostics) was used to detect apoptotic and necrotic cells by flow cytometry The method depends on the selective binding of Annexin- $\mathrm{V}$ to phosphatidylserine residues on the cell surface of apoptotic cells, and the staining by propidium iodide of cells that had lost membrane integrity. The staining procedure was performed in accordance with the procedure recommended by the manufacturer. Cells were analyzed using a FACS Calibur flow cytometer (Becton-Dickinson Biosciences). Data were analyzed using the CellQuest 3.1f software package.

\section{Statistical analysis}

The results were analyzed by two-way analysis of variance (ANOVA) with a Tukey test to determine the significance for multiple comparison. $P$-value $<0.05$ were considered statistically significant. Numerical calculations were carried out using the STATISTICA ver. 9.0 software package.

\section{Results}

\section{Apoptosis}

The percentage of apoptotic lymphocytes in the group of mice infected with $T$. spiralis (Control group) was significantly higher at 7 day after infection (dai) both in the spleen $(37.5 \%)$ and in mesenteric lymph nodes $(32.8 \%)$. The percentage of apoptotic lymphocytes in the spleen in group $\mathrm{DEX}^{+}$, was significantly higher than in the Control group from 14 dai $(16 \%, p<0.05)$ with a maximum of $34.3 \%$ at 28 dai $(p<0.001)$. For all of the other sampling times except 42 dai, the percentage of apoptotic lymphocytes was significantly higher with an average of about $18 \%$. In the spleen of mice uninfected and treated with dexametasone (group DEX) the dynamics of the population of apoptotic lymphocytes was similar to the dynamics of the lymphocyte population observed in group $\mathrm{DEX}^{+}$, but recorded values was lower (Fig. 1A).

The percentage of apoptotic lymphocytes in mesenteric lymph nodes in the group $\mathrm{DEX}^{+}$was significantly higher than in the Control group from 14 dai with a maximum of $22 \%$ at 28 dai $(p<0.001)$. In the mesenteric lymph nodes in group DEX they were less apoptotic cells than in Control group and group $\mathrm{DEX}^{+}$with exception of 35 dai (Fig. 1B). 

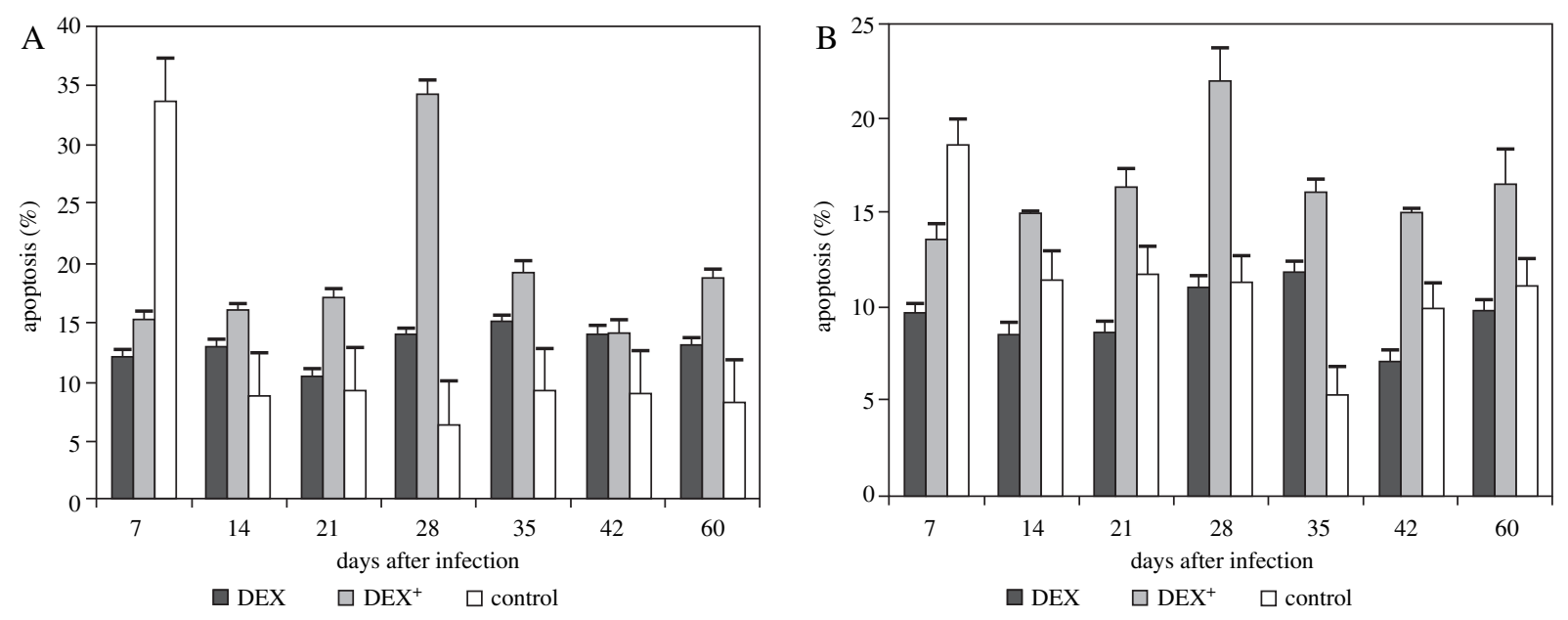

Fig. 1. Percentage of apoptotic lymphocytes: A - in the spleen, B - in mesenteric lymph nodes
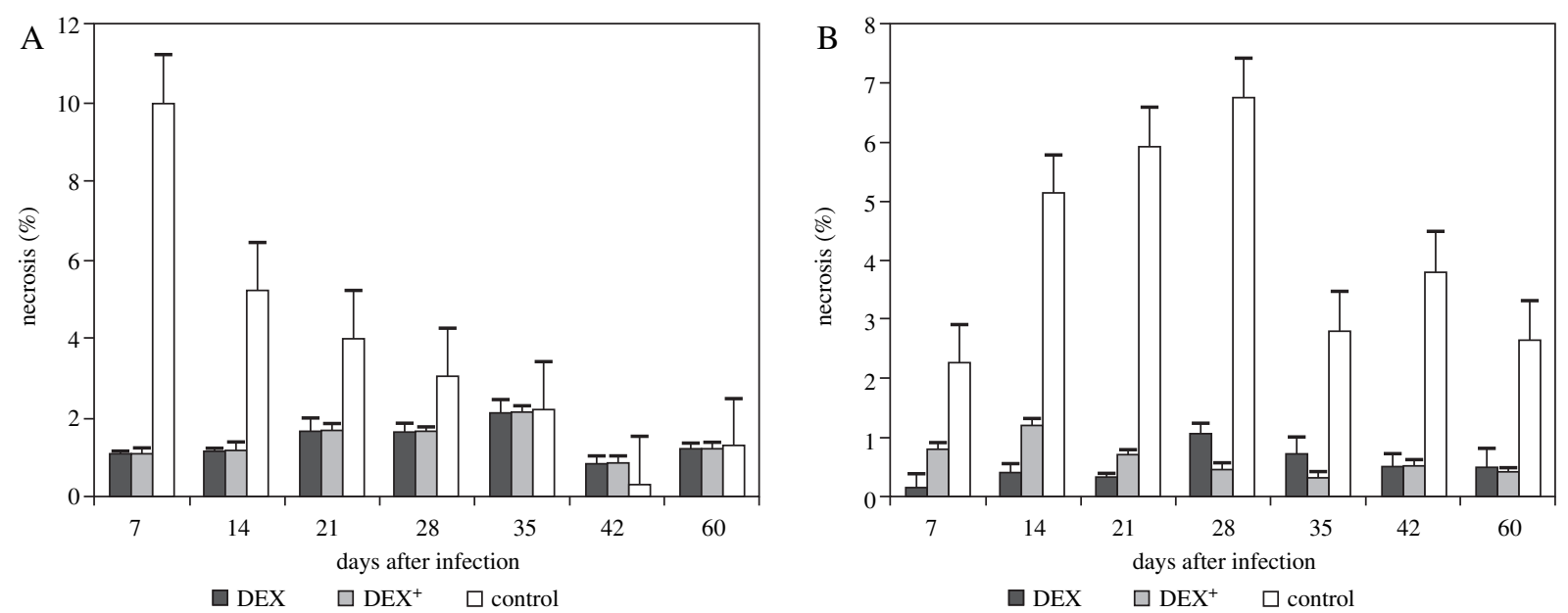

Fig. 2. Percentage of necrotic lymphocytes: A - in the spleen, B - in mesenteric lymph nodes

\section{Necrosis}

The highest percentage of necrotic lymphocytes was observed in the spleen in Control group at 7 dai $(36.1 \%)$ and at 28 dai in mesenteric lymph nodes (6.8\%) (Fig. 2A, B). The percentage of necrotic lymphocytes in the spleen in group $\mathrm{DEX}^{+}$was significantly lower than in the Control group from 7 to 28 dai. The percentage of necrotic lymphocytes in the spleen in group DEX was approximately the same as in group $\mathrm{DEX}^{+}$(Fig. 2A).

The percentage of necrotic lymphocytes in mesenteric lymph nodes in group $\mathrm{DEX}^{+}$was for all sampling times significantly lower than in the Control group. In the mesenteric lymph nodes there were no significant differences in the necrotic lymphocyte between group $\mathrm{DEX}^{+}$and group DEX (Fig. 2B).

\section{Discussion}

In the present study, the lymphocyte apoptosis and necrosis in lymphatic organs of mice infected with $T$. spiralis and treated with dexametasone was evaluated in order to asses lymphocyte response during immunosuppresion.

In contrast to necrosis, which is a passive and genetically uncontrolled process followed by an inflammatory reaction of surrounding tissue, apoptosis is an active process, which balances the cell proliferation [13]. The level of parasite infection depends on intensity of inflammatory reaction, therefore nematodes inhibits innate and adaptive proinflammatory immune response. Cytokines regulate and mediate the inflammatory response, therefore parasites try to modify the level and composition of pro- and anti-inflammatory cytokines [14]. 
The studies conducted over last few years demonstrated that parasites can induce and regulate apoptosis of $\mathrm{T}$ cells contribute to the suppression of the immune response [15]. Parasites may trigger apoptosis directly by secretion of active mediators or indirectly by producing an inflammatory milieu that promotes death of subpopulations of T lymphocytes [16-18].

Dexametasone is a glucocorticoid and acts as an antiinflammatory agent and immunosuppressant. It, like other drugs in that class, reduces the permeability of capillaries and inhibits migration of cellular elements to surrounding tissues preventing exudations and extravasations $[10,19]$. It was demonstrated that single, small doses of dexametasone administered to mice before infection with T. spiralis significantly extend the duration of the intestinal phase as well as increases infestation in muscles. Moreover, it reduces elimination of the parasites from the intestine and inhibits the response of TCD4 ${ }^{+}$lymphocytes in the intestine [20]. Dexametasone suppressed T $\mathrm{CD}^{+}, \mathrm{T} \mathrm{CD}^{+}$and $\mathrm{B}$ lymphocytes in the lamina propria of the intestinal mucosa, and $\mathrm{T} \mathrm{CD} 4^{+}$lymphocytes in inflammatory infiltrates in the muscle tissue [21]. In the previous studies conducted in mice infected with $T$. spiralis we have found that treatment with dexametasone increased the proportion of apoptotic lymphocytes in muscle tissue only at 28 days after infection, and significantly increased the proportion of necrotic lymphocytes in muscle at 21 days after infection. Moreover, the number of larvae in the muscle tissue was slightly higher in mice treated with dexametasone than in the control group [22]. In the present study in the spleen and in mesenteric lymph nodes in mice infected with $T$. spiralis and treated with dexametasone an increase in number of apoptotic cells was observed (Fig. 1A, B). The percentage of necrotic lymphocytes in both of examined lymphatic organs was weaker and less clear (Fig. 2A, B).

In earlier experiments, the stimulator of $\mathrm{T}$ cells, thymus factor X (TFX - Jelfa) was used to modulate the immune response of mice during the experimental trichinellosis [9]. Although the percentage of apoptotic lymphocytes in the spleen, mesenteric lymph nodes and muscle tissue was higher in the treated mice, this had no effect on parasite load in intestine and on the survival of parasite larvae in muscle tissue. Moreover, in mice treated with TFX, inflammatory infiltrates in muscle tissue were more extensive, and the number of inflammatory cells inside the cysts containing the larvae was higher.

Current results confirm the observation of a previous study that the extent of inflammatory exudates may be more responsible for parasite survival then percentage of lymphocytes undergoing apoptosis. Dexametasone, as it know, inhibits the activity of the cells of the immune system, including lymphocytes, by changing the composition of the cell subpopulation and by affecting the process of apoptosis.

\section{References}

1. Garside P, Kennedy MW, Wakelin D, et al. (2000): Immunopathology of intestinal helminth infection. Parasite Immunol 22: 605-612.

2. Kelly EAB, Enderson SC, Hauda KM, et al. (1991): IFN-gamma and IL-5 producing cells compartmentalize to different lymphoid organs in Trichinella spiralis-infected mice. J Immunol 147: 306-311.

3. Scott P, Kaufmann SHE (1991): The role of T-cell subset and cytokines in the regulation of infection. Immunol Today 12: 346-348.

4. Goyal PK, Hermanek J, Wakelin D (1994): Lymphocyte proliferation and cytokine production in mice infected with different geographical isolates of Trichinella spiralis. Parasite Immunol 16: 105-110.

5. Ishikawa N, Goyal PK, Mahida YR, et al. (1998): Early cytokine responses during intestinal parasitic infections. Immunology 93: 257-263.

6. Khan WI, Collins SM (2004): Immune-mediated alteration in gut physiology and its role in host defence in nematode infection. Parasite Immunol 26: 319-326.

7. James ER, Green DR (2004): Manipulation of apoptosis in the host-parasite interaction. Trends Parasitol 20: 280-287.

8. Karmańska K, Houszka M, Piekarska J (2000): The phenomenon of apoptosis in the course of experimental trichinellosis in mice. Wiad Parazytol 46: 111-115.

9. Piekarska J, Michalski A, Szczypka M, et al. (2009): Trichinella spiralis: Effect of Thymus Factor $\mathrm{X}$ on apoptosis and necrosis in mice. Exp Parasitol 123: 128-133.

10. Machnicka B, Katkiewicz M, Dziemian E, et al. (1994): Morphopathology of rats infected experimentally with Trichinella spiralis and treated with levamisole or dexamethasone. In: Trichinellosis. Ed. WC Campbell, E Pozio and F Bruschi. Istituto Superiore di Sanita Press. Rome, Italy; 427-432.

11. Kisiel K, Kaszuba A (2011): Alclometasone dipropionate properties and clinical uses. Post Dermatol Alergol 2: 107-119.

12. Fandrich F, Zhu X, Schroder J, et al. (1999). Different in vivo tolerogenicity of MHC class I peptides. J Leukocyte Biol 65: 16-27.

13. Osborne BA (1996). Apoptosis and the maintenance of homoeostasis in the immune system. Curr Opin Immunol 2: 245-254.

14. Else KJ, Finkelman FD (1998). Intestinal nematode parasites, cytokines and effector mechanisms. Int J Parasitol 28: 11451158.

15. Rutitzky LI, Mirkin GA, Stadecker MJ (2003): Apoptosis by neglect of $\mathrm{CD} 4{ }^{+}$Th cells in granulomas: a novel effector mechanism involved in the control of egg-induced immunopathology in murine schistosomiasis. J Immunol 171: 1859-1867.

16. Lundy SK, Lerman SP, Boros DL (2001): Soluble egg antigensimulated $\mathrm{T}$ helper lymphocyte apoptosis and evidence for cell death mediated by FasL ${ }^{+} \mathrm{T}$ and B cells during murine Schistosoma mansoni infection. Infect Immun 1: 271-280.

17. Lopez-Briones S, Sciutto E, Ventura JL, et al. (2003): CD4+ and CD19+ splenocytes undergo apoptosis during an experimental murine infection with Taenia crassiceps. Parasitol Res 2: $157-163$.

18. Tato P, Fernandez AM, Solano S, et al. (2004): A cysteine protease from Taenia solium metacestodes induce apoptosis in human CD4+ T-cells. Parasitol Res 92: 197-204.

19. Murosaki S, Inagaki-Ohara K, Kusaka H, et al. (1997): Apoptosis of intestinal intraepithelial lymphocytes induced by exoge- 
nous and endogenous glucocorticoids. Microbiol Immunol 41: 139-148.

20. Boźić F, Jaśarević A, Marinculić A, et al. (2000): Deksametazone as a modulator of jejunal goblet cells hyperplasia during Trichinella spiralis gut infection of mice. Helminthologia 37: 3-8.

21. Piekarska J (2003): The influence of the selected immunotrophic compounds on $\mathrm{T}(\mathrm{CD} 4+, \mathrm{CD} 8+)$ and $\mathrm{B}$ lymphocytes in the course of the experimental trichinellosis in mice. Med Weter 59: 339-343.

22. Piekarska J, Szczypka M, Michalski A, et al. (2010): The effect of immunomodulating drugs on the percentage of apoptotic and necrotic lymphocytes in inflammatory infiltration in muscle tissue of mice infected with $T$. spiralis. Pol J Vet Sci 13: 233-240. 